\title{
Half-magnetization plateau and the origin of threefold symmetry breaking in an electrically switchable triangular antiferromagnet
}

\author{
Shannon C. Haley $\odot,{ }^{1,2, *}$ Sophie F. Weber $\odot,{ }^{1,2}$ Tessa Cookmeyer $\odot,{ }^{1,2}$ Daniel E. Parker $\odot,{ }^{1}$ Eran Maniv, ${ }^{1,2}$ \\ Nikola Maksimovic $\odot,{ }_{1,2}$ Caolan John, ${ }^{1}$ Spencer Doyle $\odot,{ }^{1}$ Ariel Maniv,${ }^{3,4}$ Sanath K. Ramakrishna, ${ }^{3}$ Arneil P. Reyes, ${ }^{3}$ \\ John Singleton $\odot,{ }^{5}$ Joel E. Moore, ${ }^{1,2}$ Jeffrey B. Neaton, ${ }^{1,2,6}$ and James G. Analytis $\oplus^{1,2, \dagger}$ \\ ${ }^{1}$ Department of Physics, University of California, Berkeley, California 94720, USA \\ ${ }^{2}$ Materials Sciences Division, Lawrence Berkeley National Laboratory, Berkeley, California 94720, USA \\ ${ }^{3}$ National High Magnetic Field Laboratory, Tallahassee, Florida 32310, USA \\ ${ }^{4}$ NRCN, P.O. Box 9001, Beer Sheva, 84190, Israel \\ ${ }^{5}$ National High Magnetic Field Lab (NHMFL), Los Alamos National Laboratory (LANL), Los Alamos, New Mexico 87545, USA \\ ${ }^{6}$ Kavli Energy Nanosciences Institute at Berkeley, Berkeley, California 94720, USA
}

(Received 27 January 2020; revised 6 August 2020; accepted 11 August 2020; published 5 October 2020)

\begin{abstract}
We perform high-field magnetization measurements on the triangular lattice antiferromagnet $\mathrm{Fe}_{1 / 3} \mathrm{NbS}_{2}$. We observe a plateau in the magnetization centered at approximately half the saturation magnetization over a wide range of temperature and magnetic field. From density functional theory calculations, we determine a likely set of magnetic exchange constants. Incorporating these constants into a minimal Hamiltonian model of our material, we find that the plateau and the $\mathbb{Z}_{3}$ symmetry-breaking ground state both arise from the competition of interplane and intraplane exchange interactions. These findings are pertinent to the magnetoelectric properties of $\mathrm{Fe}_{1 / 3} \mathrm{NbS}_{2}$, which allow electrical switching of antiferromagnetic textures at relatively low current densities.
\end{abstract}

DOI: 10.1103/PhysRevResearch.2.043020

\section{INTRODUCTION}

The electrical manipulation of antiferromagnetic (AFM) spin textures has the potential to effect transformative technological change [1]. Exotic magnets with complex interactions are of special interest in this field, because they are likely to leverage novel mechanisms for their manipulation, possibly allowing ultralow power or ultrafast functionality. Diagnosing the relative magnitude of these interactions gives direct insight into these mechanisms. The existence of magnetization plateaus at fractions of saturation, when a material is subjected to large external magnetic fields, is a powerful tool to this end [2].

In this work, we study magnetization plateaus in the antiferromagnet $\mathrm{Fe}_{1 / 3} \mathrm{NbS}_{2}$, a magnetically intercalated transition metal dichalcogenide which has recently been found to exhibit reversible, electrically stimulated switching between stable magnetic states [3]. This behavior has been seen with considerably lower energy requirements in $\mathrm{Fe}_{1 / 3} \mathrm{NbS}_{2}$ as compared to the other systems [3], raising the question of whether the mechanism differs significantly [4,5]. At the center of this question is the nature of the magnetic ground state, which

\footnotetext{
*shannon_haley@berkeley.edu

†analytis@berkeley.edu
}

Published by the American Physical Society under the terms of the Creative Commons Attribution 4.0 International license. Further distribution of this work must maintain attribution to the author(s) and the published article's title, journal citation, and DOI. has been challenging to determine because collinear and noncollinear order are energetically close and the true ground state depends strongly on the magnetocrystalline anisotropy [6]. The nature of the underlying ordering in $\mathrm{Fe}_{1 / 3} \mathrm{NbS}_{2}$ has been studied by both neutron scattering [7,8] of magnetic order and optical linear birefringence microscopy [9], which probes nematic structure in the electrical conductivity. Both measurements-electric and magnetic-find indications of threefold symmetry breaking in the ground state, whose origin is unclear.

We report here a hitherto unobserved plateau in the fieldinduced magnetization at half of the saturation value. Such a plateau has been discussed theoretically $[10,11]$ in triangular lattice antiferromagnets, appearing whenever there is a significant next nearest neighbor magnetic coupling [12]. As compared to plateaus occurring at one-third saturation magnetization [13-19], experimental realizations of a halfmagnetization plateau on a triangular lattice are relatively rare $[15,20]$. The implication from theory is that the same interactions that generate the plateau are also responsible for a threefold symmetry-breaking stripe phase in the ground state, for both quantum and classical models. The halfmagnetization plateau found in $\mathrm{Fe}_{1 / 3} \mathrm{NbS}_{2}$ thus gives a clear clue regarding the microscopic mechanism for the electrically switchable antiferromagnetic ground state.

$\mathrm{Fe}_{1 / 3} \mathrm{NbS}_{2}$ is a layered material with space group $P 6_{3} 22$ no. 182 whose magnetism arises from the iron which sits between layers of $\mathrm{NbS}_{2}$ [Fig. 1(a)]. These magnetic atoms form triangular lattices in each layer, with adjacent layers staggered with respect to one another [Fig. 1(b)]. Charge from the iron atoms is transferred to the $\mathrm{NbS}_{2}$ conduction band, leaving 
(a)

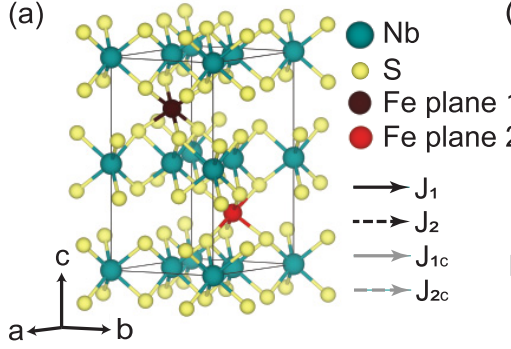

(b)

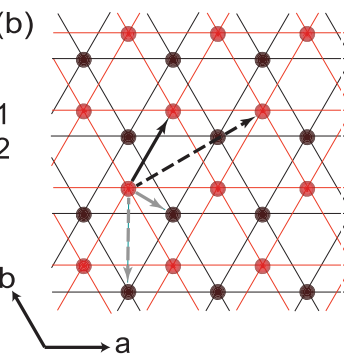

FIG. 1. (a) The crystal structure of $\mathrm{Fe}_{1 / 3} \mathrm{NbS}_{2}$. Iron atoms sit between layers of $\mathrm{NbS}_{2}$, aligned with the niobium atoms above and below. (b) Along the $c$ axis, the iron atoms in a given layer form a triangular lattice. These triangular lattices are shifted from layer to layer. Arrows indicate in-plane and out-of-plane first and second nearest neighbors, labeled by their relevant exchange constants.

them in a $2+$ ionized state, with four unpaired localized electrons per atom [21,22]. The macroscopic behavior of the material in low field is antiferromagnetic (AFM). The samples discussed in this study were grown via chemical vapor transport, as described in Ref. [23]. Using energy-dispersive x-ray spectroscopy (EDX) and inductively coupled plasma spectroscopy (ICP), the ratio of $\mathrm{Fe}: \mathrm{Nb}$ was found to be $0.330: 1$.

\section{MEASUREMENTS}

Measurements of the magnetic susceptibility as a function of temperature in low applied fields show AFM behavior below a transition near $45 \mathrm{~K}$ [Fig. 2(a)]. Fitting to the paramagnetic regime, the Curie-Weiss law yields an estimate of $5 \mu_{B} / F e$ for the effective moment of the material, a quantity which is slightly higher than the expected moment at saturation. This is in agreement with the values found in the literature, which predominantly range from 4.3 to $5 \mu_{B} / \mathrm{Fe}[7,21,24-26]$, although there is one report as high as $6.3 \mu_{B} / \mathrm{Fe}$ [27]. Heat capacity measurements resolve two clear transitions at zero field [Fig. 2(b)]. With the application of field, these transitions move apart from each other in temperature. The lower temperature transition has a further splitting at higher fields, indicating the presence of an additional intermediate phase.

High-field measurements further elucidate the nature of the phase transitions. Measurements at 0.6 and $20 \mathrm{~K}$ of the magnetization as a function of applied field are shown in Fig. 3(a). The full set of measurements, taken at temperatures ranging from 0.6 to $50 \mathrm{~K}$, is given in Appendix B, and the phase boundaries determined in part from these measurements are shown in Fig. 4. These measurements were performed on a stack of about 30 coaligned crystals, which were roughly $1 \mathrm{~mm}$ in diameter and had an average thickness of $0.1 \mathrm{~mm}$.

There are three dominant phases at low temperature evident in the data: (I) the zero-field phase characterized by a small magnetic moment, (II) the "plateau" phase characterized by a nearly constant magnetic moment centered around half the estimated saturation moment, and (III) a high-field phase which approaches the fully saturated moment. The final phase gets pushed above $60 \mathrm{~T}$ at the lowest temperatures. An intermediate phase bridging the zero-field and plateau

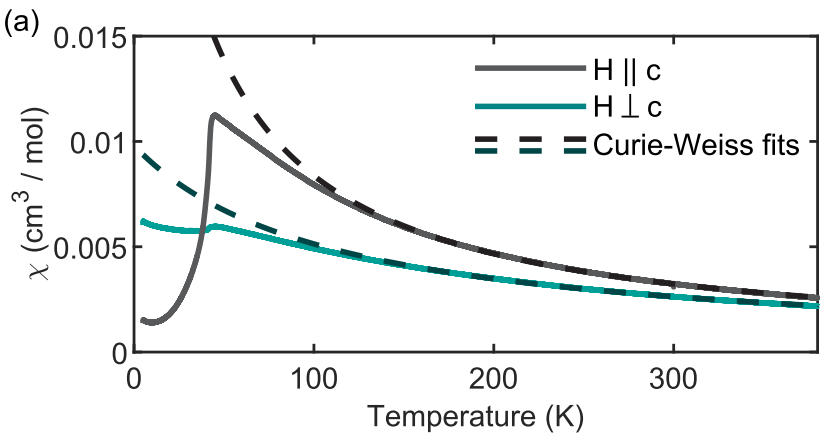

(b)

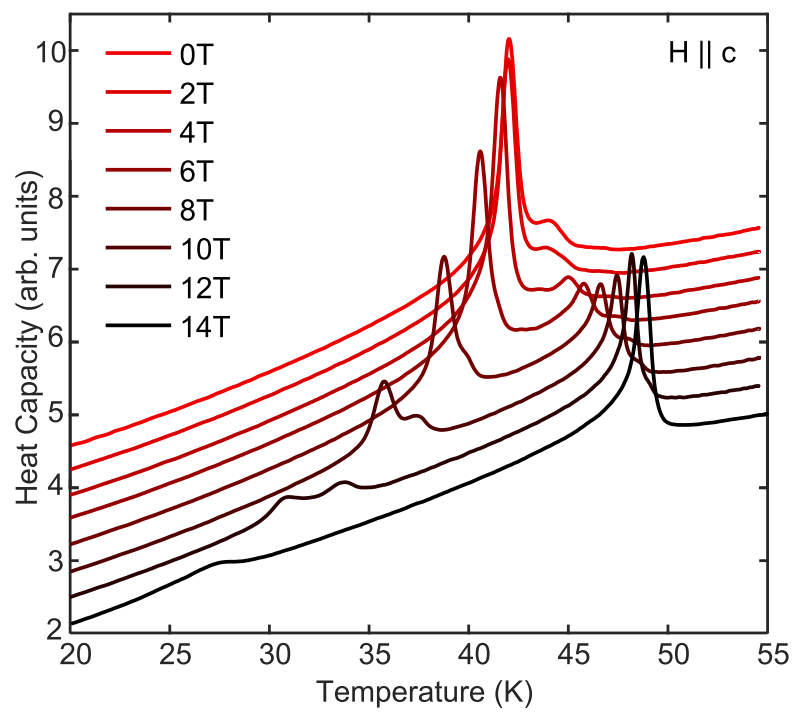

FIG. 2. (a) Curie-Weiss fits of both out-of-plane $(H \| c)$ and inplane $(H \perp c)$ susceptibility. (b) Heat capacity measurements show two transitions, which split with the application of field parallel to the $c$ axis. Curves are offset to enhance visibility.

phase has only a weak feature in the magnetization (see Appendix B).

The experimental phase diagram, Fig. 4, shows a nonmonotonic dependence of the ordering temperature on applied field. This can be explained by the impact of an applied field on a reduced dimensional system [28]; as the field increases, both the order parameter and these fluctuations are suppressed. The latter effect increases in the transition temperature in low field, and the former brings down the transition temperature at higher fields. We also observe a second ordered phase, which is destroyed in that low-field regime.

These measurements were confirmed in stacks and individual single crystals in pulsed and DC magnetic fields; see Appendix B. The latter was used to scale the former, because only relative changes could be recorded in our pulsed-field measurements. In addition, data on other compositions with $x=0.339$ suggest changes in stoichiometry do not affect the field-dependent ground state, though they can shift the phase boundaries; see Appendix B.

\section{MODEL}

To understand the physical mechanism responsible for the magnetization plateaus [Fig. 3(a)], we study a minimal model 

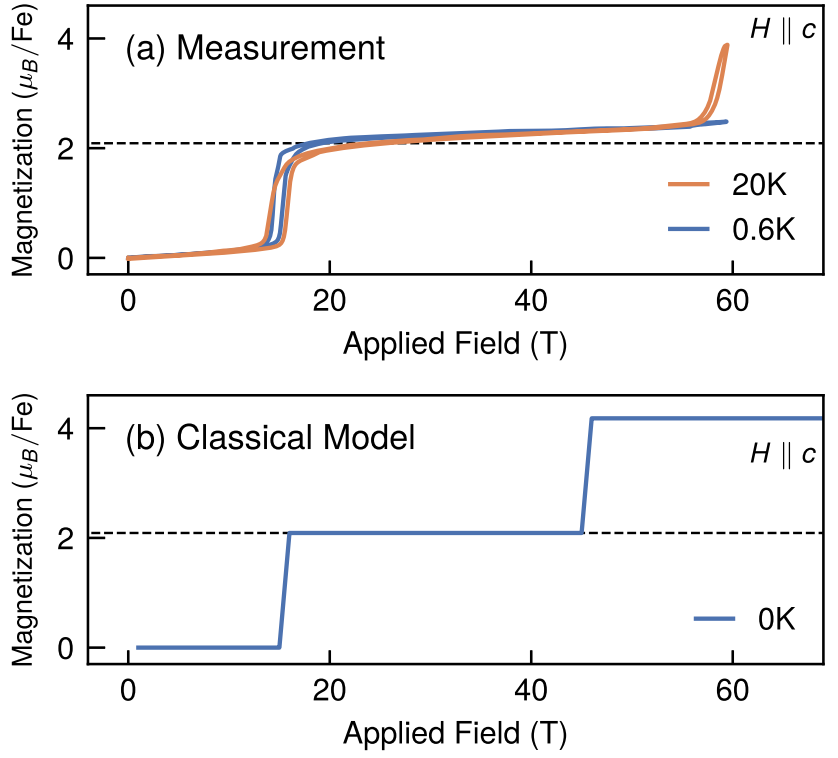

(c) Phases

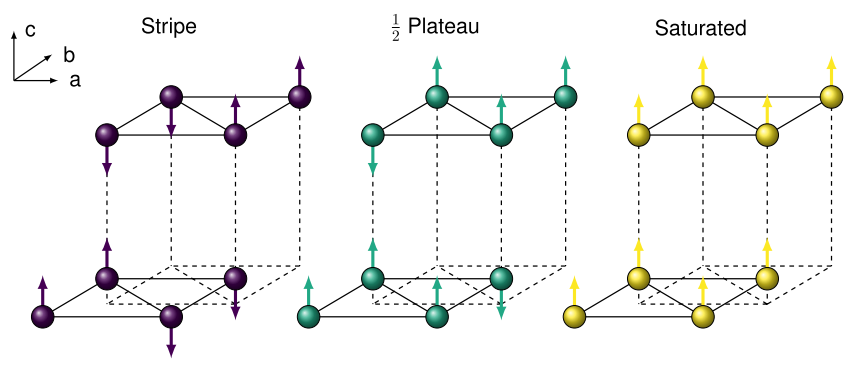

FIG. 3. (a) Magnetization response of $\mathrm{Fe}_{1 / 3} \mathrm{NbS}_{2}$ to an out-ofplane pulsed field. (Data from a 25-T pulse is used below $15 \mathrm{~T}$ for the $0.6-\mathrm{K}$ curve). At $0.6 \mathrm{~K}$, the magnetization shows two flat plateaus at 0 and $1 / 2$ of the saturated magnetization (dashed line). At $20 \mathrm{~K}$, a further transition, likely to a fully saturated state, is observed near 60 T. (b) Magnetization response of the model, Eq. (1), computed classically. Three plateaus are clearly visible: a stripy AFM phase, a UUUD phase, and a saturated PM phase. Calculational details are given in Appendix A. (c) Cartoons of the spin configurations in the eight-site unit cell.

motivated by our density functional theory (DFT) calculations, discussed below. In addition to the single-ion anisotropy $\mathrm{D}$, we find that a model with nearest neighbor (NN) and next nearest neighbor (NNN) exchange couplings within a single Fe plane, as well as NN and NNN couplings between adjacent planes, is sufficient to accurately reproduce the ab initio energies of various magnetic states. We restrict our attention to the Fe atoms and their localized $d$ states, which form a lattice of $S=2$ spins, and consider a short-range Hamiltonian

$$
\begin{aligned}
\widehat{H}= & E_{0}+2 J_{1} \sum_{\langle i, j\rangle} \widehat{\mathbf{S}}_{i} \cdot \widehat{\mathbf{S}}_{j}+2 J_{2} \sum_{\langle\langle i, j\rangle\rangle} \widehat{\mathbf{S}}_{i} \cdot \widehat{\mathbf{S}}_{j} \\
& +2 J_{1 c} \sum_{\left\langle i_{c}, j_{c}\right\rangle} \widehat{\mathbf{S}}_{i} \cdot \widehat{\mathbf{S}}_{j}+2 J_{2 c} \sum_{\left\langle\left\langle i_{c}, j_{c}\right\rangle\right\rangle} \widehat{\mathbf{S}}_{i} \cdot \widehat{\mathbf{S}}_{j}-\sum_{i} D\left(\widehat{S}_{i}^{z}\right)^{2},
\end{aligned}
$$

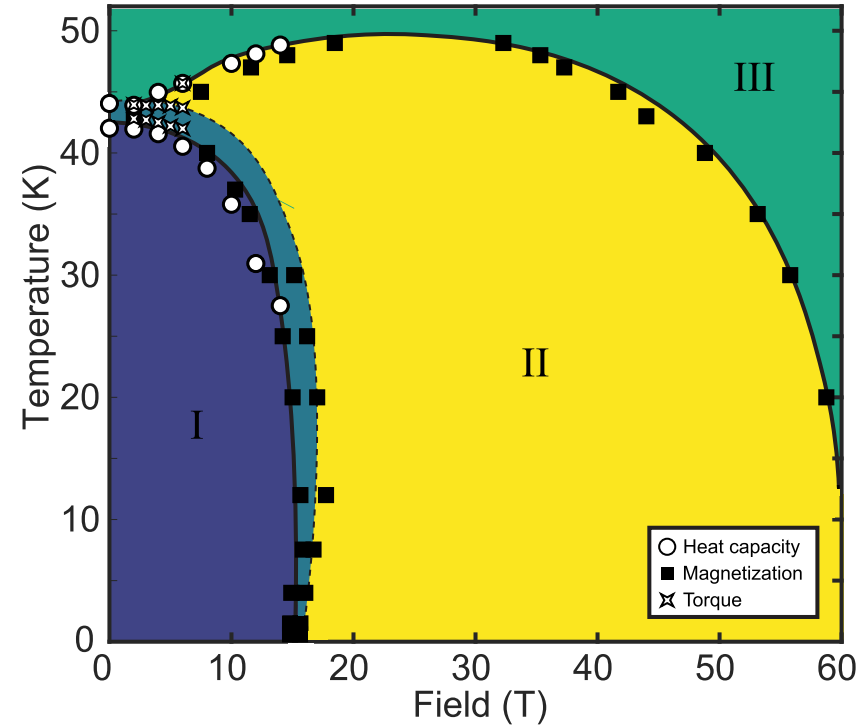

FIG. 4. Experimental phase diagram of $\mathrm{Fe}_{1 / 3} \mathrm{NbS}_{2}$, as a function of temperature and field applied along the $c$ axis. Calculations suggest that region I is a stripe phase while region II (the plateau) is UUUD. The origin of the intermediate phase bridging the stripe and UUUD phase is not known. Phase boundaries were determined by torque magnetometry, heat capacity, and pulsed field magnetization measurements; see Appendix B for details. Phase boundary lines are a guide to the eye.

where $J_{1}$ and $J_{2}$ are the NN and NNN exchange couplings within a single Fe plane, $J_{1 c}$ and $J_{2 c}$ are the NN and NNN couplings between adjacent planes, and $D$ is the magnetoanisotropy of $\mathrm{Fe}$ spins. $E_{0}$ encompasses any nonmagnetic contributions to the total energy. The exchange coupling sums are over all unique bonds. In a large neighborhood of relevant exchange coupling values, this model has three distinct phases at zero temperature as the magnetic field is varied. (1) An "AFM stripe" phase at low field with a magnetic unit cell of four Fe spins, with two pointing up along $+c$ and two along $-c$ in a stripe configuration. (2) A half-magnetization plateau at intermediate field with a magnetic unit cell of eight Fe spins, with three up spins and one down spin per layer (denoted UUUD). (3) A saturated phase at high field with a magnetic unit cell of two $\mathrm{Fe}$ spins which are all pinned to point up, parallel to $\boldsymbol{H}$. These configurations are shown in Fig. 3(c). The phases are consistent with two close antecedents of this Hamiltonian, discussed in Refs. [10,11].

Because of the spins being large $(S=2)$, we perform a classical analysis of Eq. (1). We search for the ground state of Eq. (1) using many different-sized trial unit cells. While fully three-dimensional (3D) classical Monte Carlo simulations would be more exhaustive, the present analysis is sufficient because high-field measurements of the nuclear magnetic resonance suggest that the plateau has a relatively simple spin texture; see Appendix B. We find that the magnetic unit cell for the ground state is always small over a very broad range of parameters $J, D$, and $h$, with no more than eight $\mathrm{Fe}$ atoms. Intuitively, this small unit cell is consistent with the short-ranged nature of the dominant interactions. 
TABLE I. PBE $+\mathrm{U}(U=0.3 \mathrm{eV})$ values of magnetocrystalline anisotropy $D$ and NN and NNN interplanar and intraplanar couplings in Eq. (1). Units are meV per Fe atom. With the conventions used in Eq. (1), positive values for $J$ represent AFM couplings, negative values are FM, and a positive value of $D$ implies an easy axis along $c$ for the anisotropy.

\begin{tabular}{ccccc}
\hline \hline$D$ & $J_{1}$ & $J_{2}$ & $J_{1 c}$ & $J_{2 c}$ \\
\hline 1.09 & 0.76 & -0.006 & 0.39 & -0.22 \\
\hline \hline
\end{tabular}

The classical analysis shows there is a large range of couplings $\left(J_{1}, J_{2}, J_{1 c}, J_{2 c}\right)$ which produce the three phases observed as a function of magnetic field when $D>0$ is large. The key observation is that, for $J_{1}>0$ and $J_{2} / J_{1} \ll 1$, there is a large region in the $\left(J_{1 c}, J_{2 c}\right)$ parameter space that approximately reproduces the magnetization curves-the "stripy" AFM, UUUD, and UUUU are the only three ground states for a wide range of $J_{1 c} / J_{1}>-1$ and $J_{2 c} / J_{1}<0$. In fact, the only 1/2-magnetization plateau without a UUUD structure between the two layers occurs for only a small region of parameter space. Phase diagrams are given in Appendix A. We may conclude that Eq. (1) qualitatively reproduces the observed transitions in the magnetization even without precise estimates for the coupling parameters.

We now quantitatively predict the critical magnetic fields for the transitions from the model Eq. (1). For large $D>0$, the transition from the stripe phase to the plateau phase occurs when $h=4\left(J_{1}+J_{1 c}+J_{2}\right)$ and the transition from the plateau phase to the saturated phase occurs when $h=12\left(J_{1}+J_{1 c}+\right.$ $J_{2}$ ). Quantitative analysis requires estimates of the parameters $\left(J_{1}, J_{2}, J_{1 c}, J_{2 c}, D\right)$, which we now ascertain through a combination of experimental and numerical means. Following Ref. [29], we can relate the magnetocrystalline anisotropy $D$ to the in- and out-of-plane Curie-Weiss temperatures, which are found from the fits in Fig. 2(a) to be -110 and $-26 \mathrm{~K}$, respectively; this analysis yields $D \approx 1 \mathrm{meV}$. While Ref. [21] gives slightly lower Curie-Weiss temperatures (-135 and $-40 \mathrm{~K})$, these values give a virtually unchanged estimate of $D$, which is proportional to their difference.

Our DFT calculations, performed with the Perdew-BurkeErnzerhof (PBE) functional [30] and Hubbard U corrections [31], corroborate this picture. We note that the calculated $D$, being a highly local property, is sensitive to the Hubbard $U$ used to approximately treat the localized $\mathrm{Fe} d$ electrons. This sensitivity has been documented for several Fe-based compounds in previous literature [32,33]. However, the experimental estimate of $D$ allows us to choose a $U$ value that yields a similar anisotropy and with which to compute the exchange constants in the minimal model. Using a Hubbard $U$ of $0.3 \mathrm{eV}$ in our $\mathrm{PBE}+\mathrm{U}$ calculations at experimental lattice parameters (see Appendix C for details), we obtain $D=1.09 \mathrm{meV}$, with the easy axis along $c$, in very good agreement with experiment. Using six inequivalent magnetic collinear configurations with $\mathrm{Fe}$ spins along the $c$ axis, we solve an overdetermined system of equations to determine the unknown couplings $J$. The values of all $J$ as well as $D$ are given in Table I.
As an experimental check, the Curie-Weiss temperatures can be related to the sum of the coupling constants corresponding to all of a given Fe atom's interactions, giving an estimate $\sum_{i} J_{i}=6\left(J_{1}+J_{2}+J_{1 c}+J_{2 c}\right) \approx 1.1 \mathrm{meV}$ (assuming all couplings beyond nearest and next nearest neighbors are negligible), where the factor of 6 arises because each atom has six nearest and next nearest neighbors. This is somewhat in tension with our $\mathrm{PBE}+\mathrm{U}$ results, which from Table I give $6\left(J_{1}+J_{2}+J_{1 c}+J_{2 c}\right) \approx 5.4 \mathrm{meV}$. Despite the fairly large overestimate, our $\mathrm{PBE}+\mathrm{U}$ calculations, with $U=0.3 \mathrm{eV}$ so that $D \approx 1 \mathrm{meV}$, notably yield reliable relative values of exchange constants consistent with the estimates based on our experiments. Our choice of $U$ also predicts an AFM stripy phase to have the lowest energy of all collinear magnetic configurations examined, in line with the results of our classical model and neutron data [8]. Moreover, the tendency for DFT $+\mathrm{U}$ to overestimate exchange constants at small or near-zero values of $U$ is well documented [34-36], while capturing their relative values well. Following previous work [37], we uniformly scale $J_{1}, J_{2}, J_{1 c}$, and $J_{2 c}$ so that $6\left(J_{1}+J_{2}+\cdots\right)=1.1 \mathrm{meV}$, in line with our Curie-Weiss data, and closely agreeing with the data in Ref. [21], whose fitted temperatures predict a slightly higher $\sum_{i} J_{i} \approx 1.3 \mathrm{meV}$.

Taking the scaled parameters $\left(J_{1}, J_{2}, J_{1 c}, J_{2 c}, D\right)=(0.15$, $-0.0012,0.077,-0.044,1.09) \mathrm{meV}$, we can semiquantitatively reproduce the magnetization curve. We estimate the $g$ factor as $g=2.09=g_{\mathrm{Fe}}$ [38]. This yields estimated critical fields of 15 and $45 \mathrm{~T}$, as shown in Fig. 3. With no fitting to the experimental magnetization in Fig. 3, we already have found remarkable agreement between theory and experiment. Fine-tuning the $J$ values within the range of error of the CurieWeiss data (see Appendix A) moves the transition fields into even better agreement.

\section{DISCUSSION}

The UUUD phase responsible for the half-magnetization plateau is stable at the classical level over a wide range of applied fields. The model Eq. (1) qualitatively reproduces the critical field strengths and quantitatively captures the magnitude of the magnetization. However, it fails to describe some of the fine features of the measurements, such as the small, positive slope of the magnetization within plateaus and the intermediate phase detected by measurements between the plateau and stripy order. The symmetry constraints of the switching reported in Ref. [3] also indicate an in-plane component to the moment at zero field which is not accounted for in this model. To capture the remaining fine features of $\mathrm{Fe}_{1 / 3} \mathrm{NbS}_{2}$ would require a more sophisticated 3D model with vastly more parameters and temperature effects, similar to those in Refs. [6,10]. Nevertheless, as a minimal model that only includes a subset of the degrees of freedom, the model is highly consistent with measurements and seems to have identified the dominant interactions responsible for the magnetization response of $\mathrm{Fe}_{1 / 3} \mathrm{NbS}_{2}$.

The applicability of the lattice model suggests that $\mathrm{Fe}_{1 / 3} \mathrm{NbS}_{2}$ is proximate to many other phases, some of which are possibly similar to supersolid phases discussed by Seabra and Shannon [10]. One of these may describe the boundary 
phase dividing stripy and plateau orders in Fig. 4. Preliminary data appears to be consistent with an UUD phase (see Appendix B), though more data are required to confirm this.

The agreement of the experimentally observed magnetization with a classical model suggests that the magnetic behavior, while originating from many competing interactions, involves conventional magnetic phases. This model could be further confirmed by inelastic neutron scattering. The existence of an UUUD half-magnetization plateau had previously been studied as a result of strong next nearest neighbor interactions within the triangular-lattice plane; we have determined that it is not limited to that case, as we see its emergence from strong interplanar interactions. The determination of these interactions and of the abnormally strong single-ion anisotropy has a large impact on the zero-field ground state of this material; the threefold symmetry breaking seen in optical measurements [9], for example, originates from a magnetic order driven by a large ratio of $J_{1 c} / J_{1} \approx 1 / 2$, likely stripy in nature with a significant $c$-axis component. It is interesting to consider the implications for the electrical switching of the spin texture of this material. In the typical mechanism, an in-plane Néel vector can be naturally rotated by the angular momentum imparted by an in-plane spin polarized current. In contrast, $\mathrm{Fe}_{1 / 3} \mathrm{NbS}_{2}$ has a Néel vector that is predominantly pointed out-of-plane, so that a different kind of mechanism to transfer angular momentum is likely to be active. The present work suggests that this leverages both strong interand intraplanar exchange interactions.

\section{ACKNOWLEDGMENTS}

This work was supported as part of the Center for Novel Pathways to Quantum Coherence in Materials, an Energy Frontier Research Center funded by the US Department of Energy, Office of Science, Basic Energy Sciences. Work by J.G.A. and S.C.H. was funded in part by the Gordon and Betty Moore Foundation's EPiQS Initiative, Grant No. GBMF9067 to J.G.A. Work by T.C. and D.E.P. was supported by NSF Graduate Research Fellowship Program, NSF DGE No. 1752814. A portion of this work was performed at the National High Magnetic Field Laboratory, which is supported by National Science Foundation Cooperative Agreement No. DMR-1644779 and the State of Florida.

\section{APPENDIX A: CLASSICAL ANALYSIS OF THE SPIN MODEL}

This Appendix will analyze the proposed minimal spin model at the classical level. We will show the following:

(1) A magnetic unit cell with six or eight spins is favored for all reasonable parameter values.

(2) There is a wide range of parameters where the three dominant phases of the model are, as a function of applied field,

(a) AFM stripy,

(b) 1/2-plateau, and

(c) saturated.

(3) However, there are a number of "nearby" phases in parameter space-particularly a 1/3-plateau-which could appear for small parameter ranges.

For reference, let us recall the model from the main text. We consider a minimal model for the magnetic degrees of freedom that are relevant at low temperature: the two iron atoms per unit cell, which are known to be in the $\mathrm{Fe}^{2+}$ state, which has $S=2$. DFT calculations indicated that the most important couplings are the single-ion anisotropy $D$, the in-plane nearest neighbor (NN) and next nearest neighbor couplings (NNN) $J_{1}$ and $J_{2}$ respectively, and their out-of-plane counterparts $J_{1 c}$ and $J_{2 c}$. As one can see from Fig. 1 of the main text, each Fe atom has six neighbors of each of the four types. In a magnetic field, the minimal model Hamiltonian is

$$
\begin{aligned}
\widehat{H}= & E_{0}+2 J_{1} \sum_{\langle i, j\rangle} \widehat{\mathbf{S}}_{i} \cdot \widehat{\mathbf{S}}_{j}+2 J_{2} \sum_{\langle\langle i, j\rangle\rangle} \widehat{\mathbf{S}}_{i} \cdot \widehat{\mathbf{S}}_{j}+2 J_{1 c} \sum_{\left\langle i_{c}, j_{c}\right\rangle} \widehat{\mathbf{S}}_{i_{c}} \cdot \widehat{\mathbf{S}}_{j_{c}}+2 J_{2 c} \sum_{\left\langle\left\langle i_{c}, j_{c}\right\rangle\right\rangle} \widehat{\mathbf{S}}_{i_{c}} \cdot \widehat{\mathbf{S}}_{j_{c}} \\
& -\sum_{i} D\left(\widehat{S}_{i}^{z}\right)^{2}-S \boldsymbol{h} \cdot \sum_{i} \widehat{\mathbf{S}}_{i},
\end{aligned}
$$

where $\boldsymbol{h}=\mathbf{H} \mu_{B} g / S$ is the external field and the sums are over all unique pairs $i, j$ that are $\mathrm{NN}, \mathrm{NNN}$, and $\mathrm{NN}$ between layers, and NNN between layers, respectively. Finally, $E_{0}$ stands for the energy of all nonmagnetic degrees of freedom (essentially the electronic ground-state energy).

We now proceed to analyze this model. In principle, this is a quantum model. However, since the spin is quite large $(S=2)$, the first terms in the $1 / S$ expansion should be almost exact. Therefore, we will work entirely at the classical level.

\section{The magnetic unit cell is small}

To begin the analysis, we first find the magnetic unit cell. Ordinarily one would do this by classical Monte Carlo methods and measuring the size of the unit cell. However, the model is straightforward enough that this is unnecessary. As all the interactions are short ranged, we expect the unit cell to be quite small.

To verify this, we perform an extensive search of possible magnetic unit cells up to 16 atoms. For each candidate magnetic unit cell, we find the classical ground state via global optimization using the basin-hopping algorithm. Since the phase spaces in question are relatively low-dimensional $(2 \times 16$ at most $)$, this is a fast and reliable way to find the true ground state. We also tried several other global minimization algorithms, which yielded identical results, showing that basin hopping is robust.

By sampling parameter space $\left(J_{1}, J_{2}, J_{1 c}, J_{2 c}\right)$ at a broad range of points and $D \approx 1 \mathrm{meV}$, we may conclude the following. First, all classical ground states have a magnetic unit cell 
with either six or eight atoms: three or four atoms in a planar layer and two layers. There are actually two relevant unit cells of size 8 , one tiling the plane in a rectangular lattice and the other as a triangular lattice. Second, we find all ground-state spin textures have spins along the $c$ axis. This is expected because the anisotropy is quite large; we are in the Ising limit of Eq. (A1). Third, we identify a total of 14 classical ground states for this model across parameter space, which we refer to as states $A$ through $M$. Their spin textures are shown in Fig. 9. We will examine the phase diagram below and see there is a large region of parameter space in which one has AFM stripy, 1/2-plateau, and saturated phases in order as one increases the magnetic field. For now, we may conclude that the magnetic unit cell has six or eight atoms and examine its energetics and transitions more carefully.

\section{Candidate phases and transitions}

The small magnetic unit cells identified above are relatively simple and we can write analytic expressions for the energies of many phases and some of the phase boundaries. When the Hamiltonian is restricted to one of the three small magnetic unit cells (the six-atom unit cell, the eight-atom unit cell with triangular tiling, and the eight-atom unit cell with rectangular tiling), the energy may be written as

$$
\begin{aligned}
& = \begin{cases}4\left(J_{1 c}+J_{2 c}\right)\left(\mathbf{S}_{\ell 1} \cdot \mathbf{S}_{\ell 2}\right)+2 J_{1}\left(\frac{3}{2}\left[\left(\mathbf{S}_{\ell 1}\right)^{2}+\left(\mathbf{S}_{\ell 2}\right)^{2}-6 S^{2}\right]\right)+36 J_{2} S^{2} & \text { six-spin unit cell } \\
2\left(J_{1}+J_{2}\right)\left(\left(\mathbf{S}_{\ell 1}\right)^{2}+\left(\mathbf{S}_{\ell 2}\right)^{2}-8 S^{2}\right)+4 J_{1 c}\left(\mathbf{S}_{\ell 1} \cdot \mathbf{S}_{\ell 2}\right) & \text { eight-spin unit cell with triangular tiling } \\
\quad+\left(12 J_{2 c}-4 J_{1 c}\right)\left(\mathbf{S}_{1} \cdot \mathbf{S}_{\tilde{4}}+\mathbf{S}_{2} \cdot \mathbf{S}_{\tilde{1}}+\mathbf{S}_{3} \cdot \mathbf{S}_{\tilde{2}}+\mathbf{S}_{4} \cdot \mathbf{S}_{\tilde{3}}\right) & \\
2\left(J_{1}+J_{2}\right)\left(\left(\mathbf{S}_{\ell}\right)^{2}\right. & \end{cases} \\
& \frac{E}{N_{u}}=\left\{\begin{array}{c}
2\left(J_{1}+J_{2}\right)\left(\left(\mathbf{S}_{\ell 1}\right)^{2}+\left(\mathbf{S}_{\ell 2}\right)^{2}\right)-16 J_{1} S^{2}+4 J_{1 c}\left(\mathbf{S}_{\ell 1} \cdot \mathbf{S}_{\ell 2}\right) \\
-8 J_{2}\left(\mathbf{S}_{1} \cdot \mathbf{S}_{3}+\mathbf{S}_{2} \cdot \mathbf{S}_{4}+\mathbf{S}_{\tilde{1}} \cdot \mathbf{S}_{\tilde{3}}+\mathbf{S}_{\tilde{2}} \cdot \mathbf{S}_{\tilde{4}}\right)
\end{array}\right. \\
& +\left(8 J_{2 c}-4 J_{1 c}\right)\left(\mathbf{S}_{1} \cdot \mathbf{S}_{\tilde{4}}+\mathbf{S}_{2} \cdot \mathbf{S}_{\tilde{3}}+\mathbf{S}_{3} \cdot \mathbf{S}_{\tilde{2}}+\mathbf{S}_{4} \cdot \mathbf{S}_{\tilde{1}}\right) \\
& +4 J_{2 c}\left(\mathbf{S}_{1} \cdot \mathbf{S}_{\tilde{2}}+\mathbf{S}_{2} \cdot \mathbf{S}_{\tilde{1}}+\mathbf{S}_{3} \cdot \mathbf{S}_{\tilde{4}}+\mathbf{S}_{4} \cdot \mathbf{S}_{\tilde{3}}\right) \\
& -D \sum_{j=i, \tilde{i}}\left(\widehat{S}_{j}^{z}\right)^{2}-\boldsymbol{h} \cdot \sum_{j=i, \tilde{i}} \mathbf{S}_{j}, \\
& \mathbf{S}_{\ell 1 / \ell 2}=\sum_{j=i / \tilde{i}} \mathbf{S}_{j}
\end{aligned}
$$

where $\tilde{i}$ is in one layer and $i$ is in a different layer, the sums are over the unit cell, and $\mathbf{S}_{\ell 1 / \ell 2}$ are the sums of the spins within layers 1 and 2 respectively. $N_{u}$ stands for the number of unit cells, so the energy per atom would require dividing by six or eight depending on the case. The tiling refers to whether to shift the unit cell along the cardinal directions or along the directions of the bases of the triangular lattice, as discussed in the caption of Fig. 5 .

When $D$ is taken large enough for the spins to align along the $c$ axis, we can use (A2) to find explicit expressions for the energies of various spin configurations. Focusing on the four phases seen in the experiment-an AFM "stripy" ground state, a possible 1/3-magnetization phase, a 1/2magnetization plateau phase, and a paramagnetic phase-in a magnetic field $\boldsymbol{h}=h \hat{c}$, we find

$$
\frac{E}{N S^{2}}+D=\left\{\begin{array}{ll}
6 J_{2 c}-2\left(J_{1}+J_{2}+J_{1 c}\right) & \text { AFM stripy } \\
\frac{2}{3}\left(J_{1 c}+J_{2 c}\right)-2 J_{1}+6 J_{2}-\frac{1}{3} h & 1 / 3 \text {-plateau } \\
6 J_{2 c}-\frac{1}{2} h & 1 / 2 \text {-plateau } \\
6\left(J_{1}+J_{2}+J_{1 c}+J_{2 c}\right)-h & \text { paramagnetic }
\end{array},\right.
$$

where $N$ is the number of atoms in the system, and the exact way the spins are arranged is seen in Fig. 9.

The most clear transitions seen in the material are from AFM stripy to 1/2-plateau at $h_{c 1}$ and from 1/2-plateau to paramagnetic at $h_{c 2}$. We can solve for the critical fields of the transition and see

$$
\frac{1}{3} h_{c 2}=h_{c 1}=4\left(J_{1}+J_{1 c}+J_{2}\right) .
$$

To compare directly to experiment, we use the experimentally supported value $g=g_{\mathrm{Fe}}=2.09$ [38]. This yields a

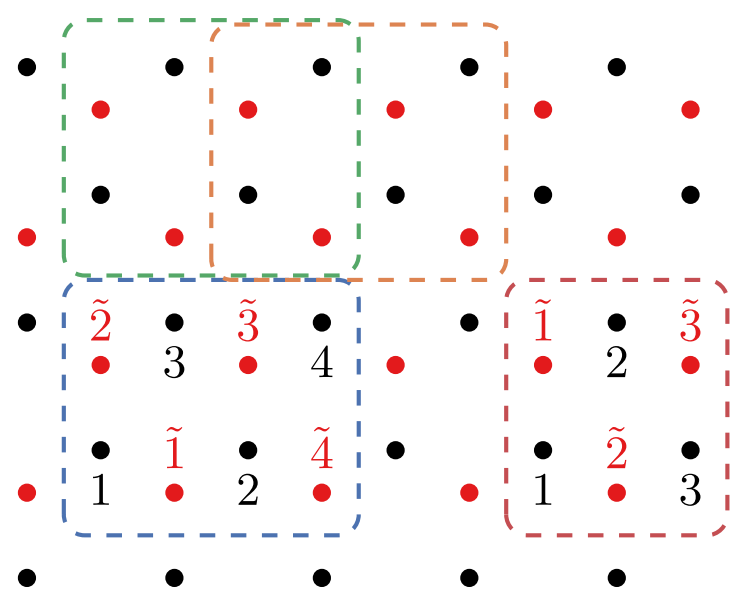

FIG. 5. A figure showing the lattice convention used in Eq. (A2). The eight-spin unit cell is seen on the left and the six-spin unit cell is seen on the right. Triangular tiling refers to shifting the blue unit cell to the orange unit cell (as well as to the right) to generate the lattice. Rectangular tiling refers to shifting the blue unit cell to the green unit cell (as well as to the right). The $\tilde{i}$ 's refer to the second layer as seen in Fig. 1 in the main text. 


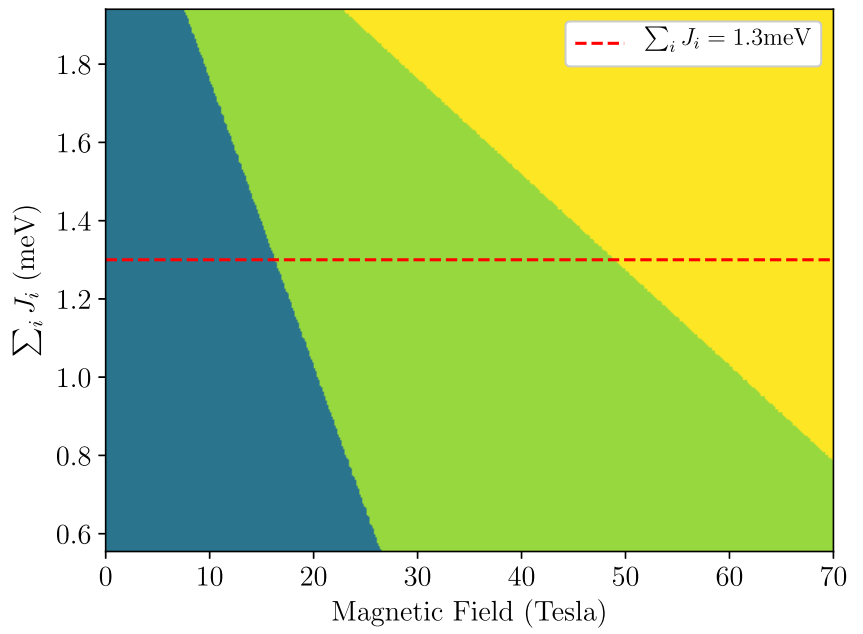

FIG. 6. The phase diagram of Eq. (A1) as a function of applied field when the ratios between the $J$ 's are given from DFT but the overall sum $\sum_{i} J_{i}$ is allowed to vary. The phases from left to right are AFM stripy, 1/2-plateau, and saturated. The dashed red line corresponds to the magnetization profile shown in Fig. 3 of the main text. One can see that changing $\sum_{i} J_{i}$ changes the location of the phase boundaries but does not qualitatively change the magnetization response.

critical field of

$$
\frac{1}{3} H_{c 2}=H_{c 1}=\frac{S}{g \mu_{B}} h_{c 1} \approx(66 \mathrm{~T} / \mathrm{meV})\left(J_{1}+J_{1 c}+J_{2}\right) .
$$

Using the raw values from DFT given in Table I of the main text, this would give transitions at $H_{c 1}=77 \mathrm{~T}$ and $H_{c 2}=$ $226 \mathrm{~T}$. However, as discussed in the main text, there is strong evidence that DFT overestimates the absolute values of the $J$ 's dramatically but has the correct relative values. If we rescale the DFT parameters to match the experimental Curie-Weiss results, as discussed in the main text, then we find

$$
\begin{aligned}
& H_{c 1} \approx 15 \mathrm{~T}, \\
& H_{c 2} \approx 45 \mathrm{~T},
\end{aligned}
$$

which are fairly comparable with the experimental results (shown in Fig. 3 of the main text). It is worth noting that shifting the phase boundaries by $5 \mathrm{~T}$ requires a change in the coupling constants of $<0.1 \mathrm{meV}$, as shown in Fig. 6, so it is likely that weak longer range couplings we have neglected in this minimal model, or a relatively small amount of error in the Curie-Weiss estimate, could shift the transition to more closely match the experiment.

\section{3. $J_{1}-J_{1 c}-J_{2 c}$ phase diagrams}

We now examine the phase diagram of the spin Hamiltonian (A1). We shall see that there is a large neighborhood of parameter values which reproduces the phases found in the experiment.

We take $D$ large to ensure the spins are aligned with the field. To start, we set $J_{1}=1, J_{2}=0$ and allow $J_{1 c}$ and $J_{2 c}$ to vary. The phase diagram in $\left(J_{1 c}, J_{2 c}\right)$ space is shown in Fig. 7 for zero magnetic field. One can see that there is a large neighborhood around the DFT values where the classical

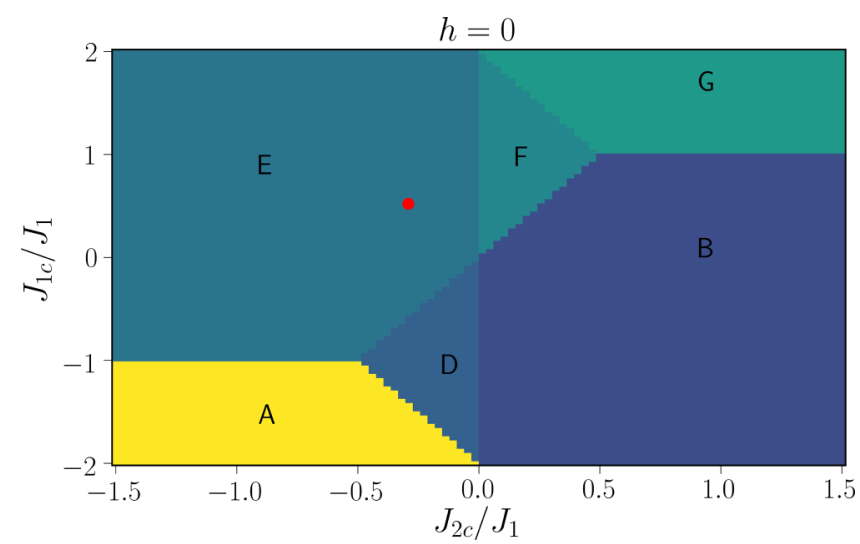

FIG. 7. The phase diagram of Eq. (A1) for $h=0$ when $\left|J_{2} / J_{1}\right| \ll$ $1, J_{1}>0$, and $D$ large enough to ensure the spins are aligned to the $c$ axis. The exchange parameters from DFT are shown as a red dot. The spin configurations for each color are seen in Fig. 9. Since the experiment finds AFM order, the ground state cannot be phase A (a ferromagnetic ordering). When we look at field dependence in Fig. 8, in order for there to only be three phases with an intermediate $1 / 2$ magnetization plateau phase, the ground state must either be phase $\mathrm{E}$ or phase B, which are both AFM stripe phases differing only in how the layers are stacked.

ground state is AFM stripy, consonant with experiment. We now allow the magnetization to vary. Figure 8 shows phase diagrams for $\left(h, J_{1 c}\right)$ for a variety of $J_{2 c}$ values. Many magnetic

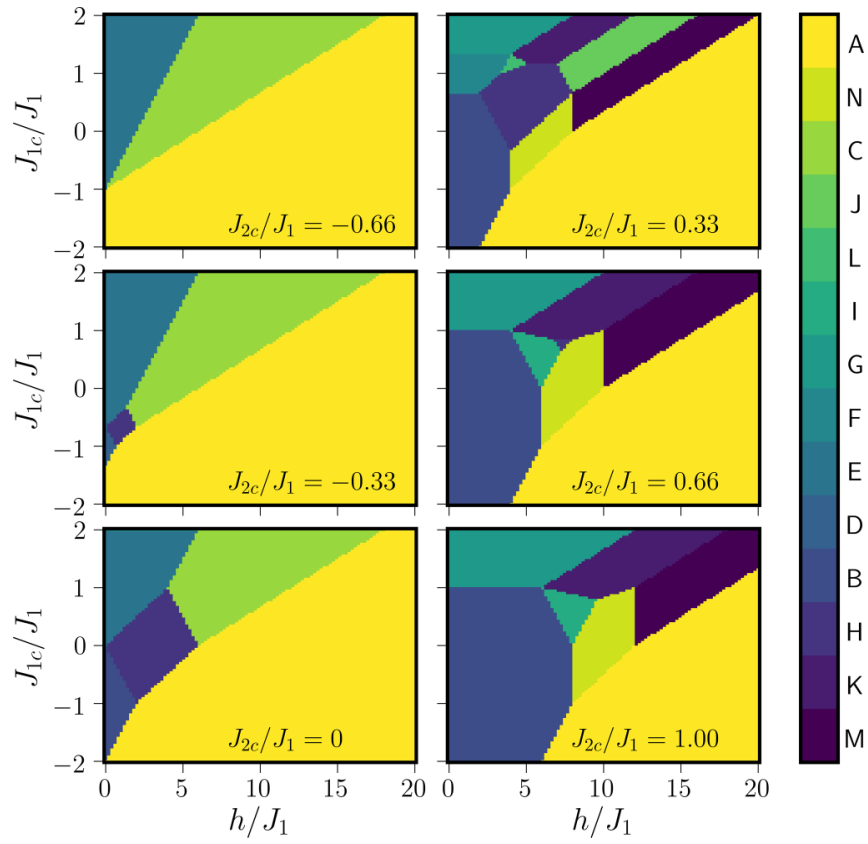

FIG. 8. The phase diagram of Eq. (A1) when $\left|J_{2} / J_{1}\right| \ll 1, J_{1}>$ 0 , and $D$ large enough to ensure the spins are aligned to the $c$ axis. The spin configurations for each color are seen in Fig. 9. It is clear for a wide range of $J_{1 c} / J_{1}>-1$ and $J_{2 c}<0$ the three phases as field increases-AFM stripy, UUUD in each layer, and paramagnet-are always seen. Phases H, K, and M (that are colored purple) are sixspin unit cells, whereas the other phases have eight-spin unit cells. The DFT estimates of the parameters yield $J_{2 c} / J_{1} \approx-0.29$. 


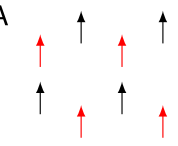

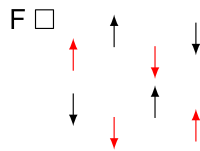

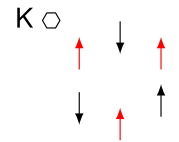

B

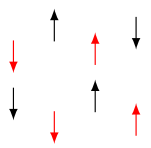

G
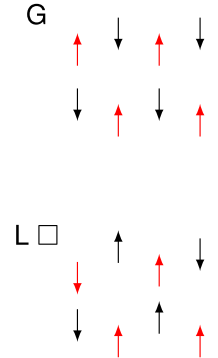

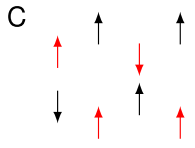

$\mathrm{HO}$

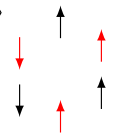

$\mathrm{M}$

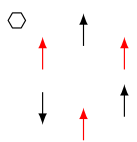

$\mathrm{D} \square$
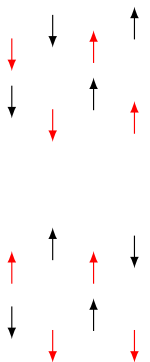

$\mathrm{N}$

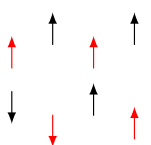

FIG. 9. The arrangements of the spins for all the phases in Fig. 7. A $\square$ next to the phase name indicates that it has a rectangular tiling whereas the absence of a $\square$ indicates a triangular tiling. A hexagon indicates a six-spin configuration. The DFT parameters, as shown in Fig. 7, have phase $\mathrm{E}$ as the ground state and phase $\mathrm{C}$ as the intermediate plateau phase. Phase $\mathrm{A}$ is always the saturated phase.

phases emerge, as indicated by the color, and the different configurations are shown in Fig. 9.

It is clear in a large neighborhood of the DFT parameters that the basic phase diagram is the same: There is a stripy phase (phase E) which transitions to the UUUD phase (phase C) at higher field and there is a transition to a PM phase (phase A) at the largest fields. The relative DFT values for the couplings, scaled down to match the experimental estimate, are therefore quite reasonable.

We should mention that there is some debate in the literature about whether the ground state is a stripe or zigzag phase (B, E and D, F respectively) [7,8]. From our DFT calculations,
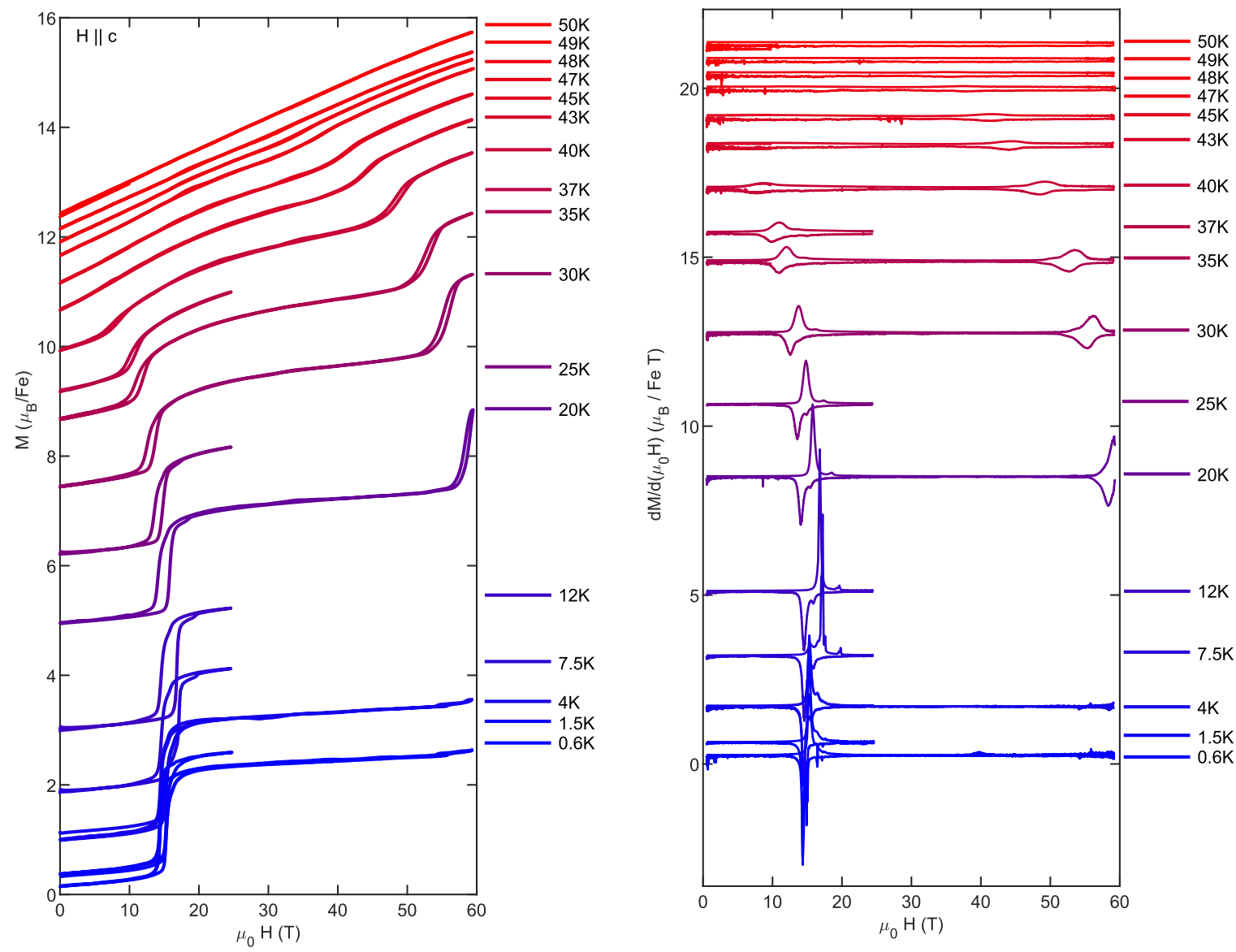

FIG. 10. High-field measurements of the out-of-plane magnetization (left) and susceptibilities (right), taken at a variety of temperatures. 
FexNbS2, $x=0.33$

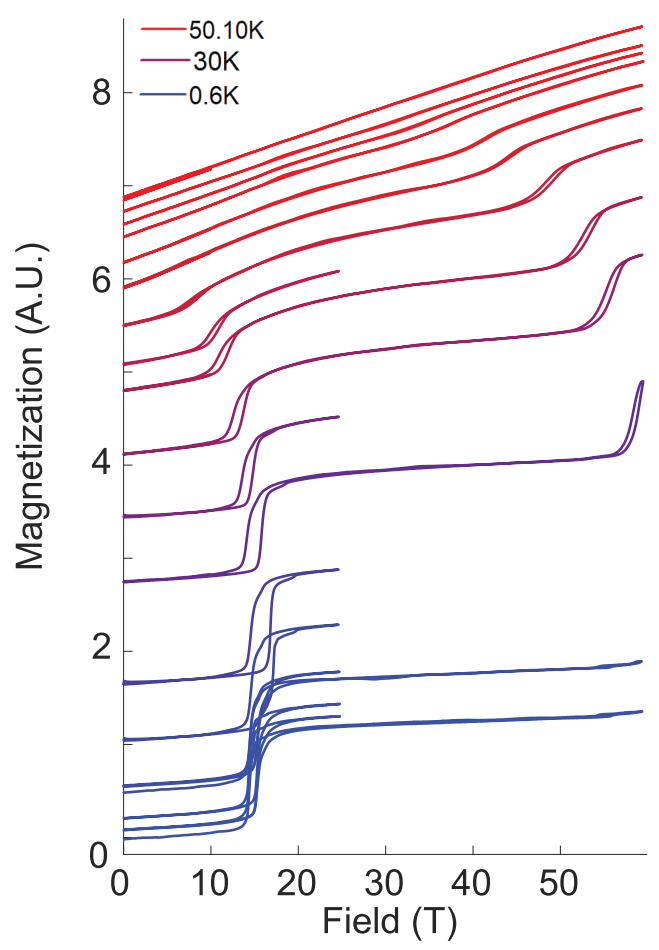

FexNbS2, $x=0.339$

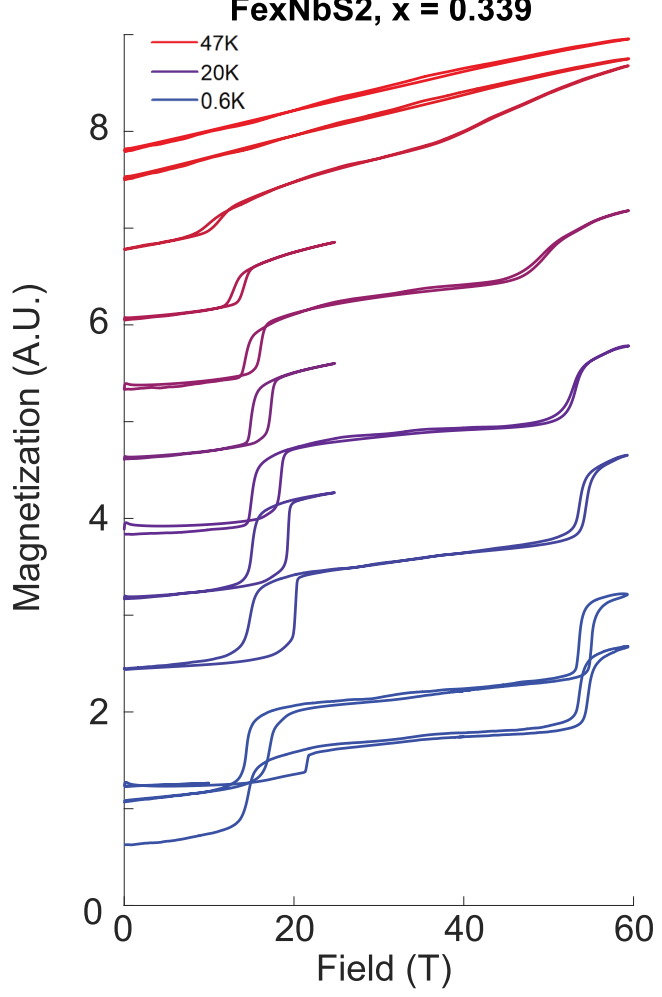

FIG. 11. A side-by-side comparison of the pulsed field magnetization of $\mathrm{Fe}_{0.33} \mathrm{NbS}_{2}$, left, and $\mathrm{Fe}_{0.339} \mathrm{NbS}_{2}$, right.

by comparing total energies of all four phases we find that, consistent with Ref. [8], the AFM stripe (E) is lower in energy than both zigzag phases and the FM stripe (B) (FM stripe B differs from $\mathrm{E}$ only in the stacking of the Fe planes). This is also consistent with the prediction of the ground state found by plugging in the DFT values of Heisenberg couplings into our model Hamiltonian. Moreover, the zigzag phases D and F do not reproduce the simple three-phase structure as a function of magnetic field that we observe experimentally.

In the experiment, the potential appearance of a state with a $1 / 3$-plateau suggests that the exact parameter choice lies near the boundary of the 1/3-plateau phase and the 1/2-plateau phase, which can be achieved by lowering $\left|J_{1 c}\right|,\left|J_{2 c}\right|$, or both from their current estimate from DFT.

These phase diagrams show that our minimal model Eq. (A1) qualitatively reproduces the magnetization response for a wide range of parameter values and is therefore consistent with experiment. So, even though we do not have highly accurate estimates for the $J$ 's, this is evidence that Eq. (A1) is a good minimal model for the magnetic degrees of freedom in $\mathrm{Fe}_{1 / 3} \mathrm{NbS}_{2}$. To improve this model, one would have to include longer range couplings and perhaps include other types of spin interactions, such as a biquadratic term. Furthermore, one could include postclassical corrections, which would likely modify the magnetization response slightly, shift the phase boundaries, and possibly stabilize addition phases.

\section{APPENDIX B: ADDITIONAL MEASUREMENTS}

This section provides a number of additional and unabridged measurements.

\section{High-field magnetization}

The magnetization as a function of applied field was measured in pulsed magnetic field, with several 60- and 25-T measurements, shown in Fig. 10. The susceptibility, as determined by taking the field derivative of the magnetization data, is shown as well for ease of identifying phase transitions. The phase boundaries in the experimental phase diagram (Fig. 4 in the main text) are the centers of the hysteresis loops, calculated as the midpoint between the peaks in susceptibility on the way up ( 0 to $60 \mathrm{~T})$ and on the way down (60 to $0 \mathrm{~T}$ ). The $60-\mathrm{T}$ measurements taken at 0.6 and $4 \mathrm{~K}$ have a discontinuity where the gain was saturated due to the magnetization changing too quickly during the 0 - to $60-\mathrm{T}$ leg of the pulsed measurement. Additional measurements up to $25 \mathrm{~T}$ were taken at those temperatures, which did not have the same saturation issue. The $0.6-\mathrm{K}$ measurement found in the text is predominantly from the 60-T pulse, with the saturated portion of the measurement replaced with the data from the 25-T pulse. The 0- to 60-T leg of the measurement is entirely from the 60-T pulse.

\section{DC field and single-crystal measurements}

The primary results presented in this paper are based on 60-T pulsed field measurements of a stack of 30 coaligned crystals all from the same growth batch. The nature of these results, including the sharpness and location of the transition, was confirmed via 30-T DC field measurements of the stack [Fig. 13(a)] and 60-T pulsed field measurements of a single crystal [Fig. 13(b)]. The center of the phase transition does not move with the slower sweep rate of a DC measurement, although it does broaden slightly. There is strong agreement 

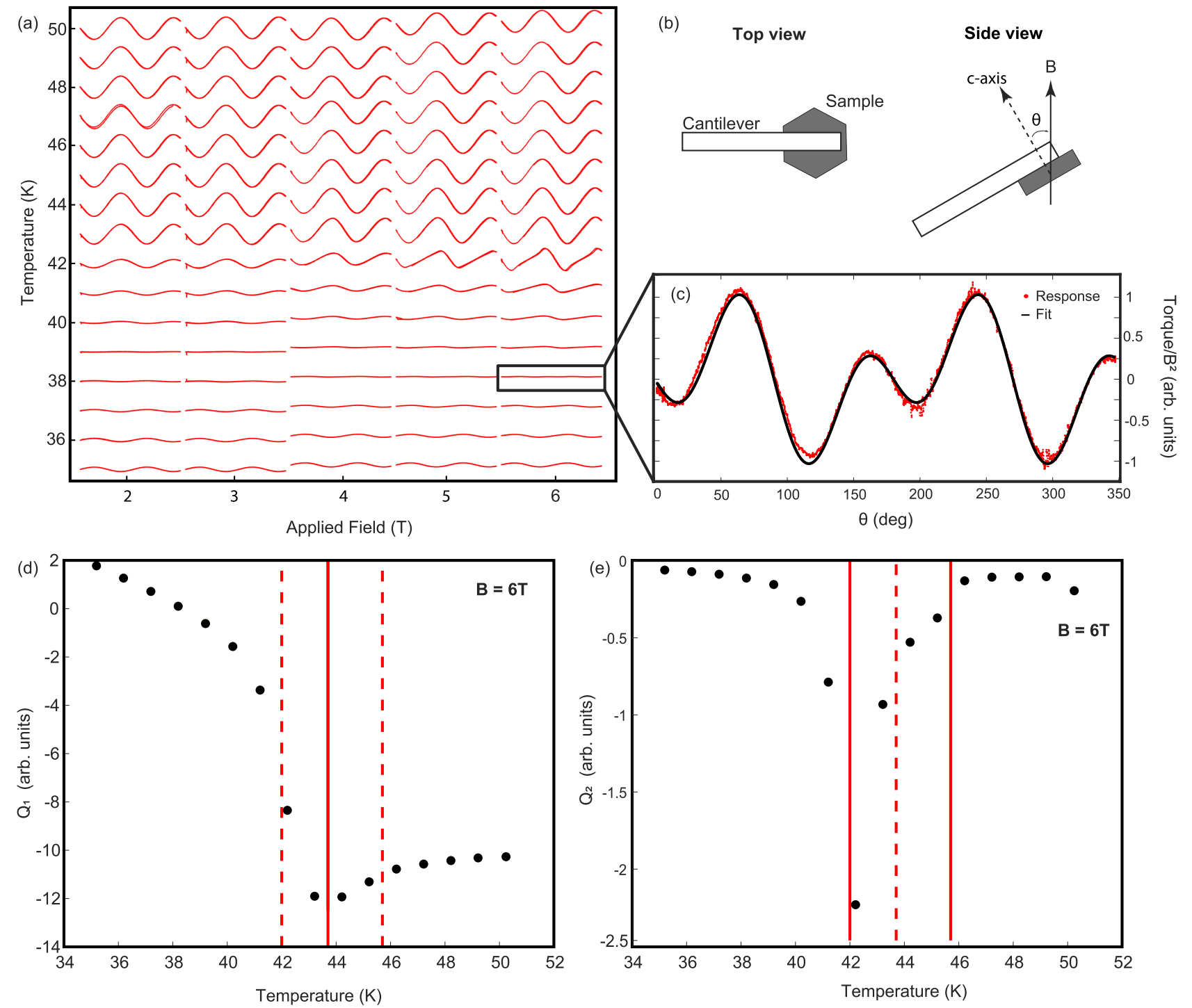

FIG. 12. (a) Torque-vs-angle curves at temperatures from 35 to $50 \mathrm{~K}$ and fields from 2 to $6 \mathrm{~T}$. The curves shown are normalized to the field squared, but this is not important for the analysis, which compares measurements taken at the same field. (b) Schematic of the experiment, establishing the relevant directions and meaning of the angle $\theta$. (c) An example of a response curve shown with its fit. This measurement was taken at $38.2 \mathrm{~K}$ and $6 \mathrm{~T}$. [(d), (e)] The amplitudes of the $\sin 2 \theta$ and $\sin 4 \theta$ components, respectively, of the response curves as a function of temperature. A solid red vertical line indicates the identification of a transition from the given plot. A dotted red vertical line indicates the location of a transition which was identified from the other plot.

between the pulsed field single and stacked crystal curves. This shows that the location of the transition is not sample dependent and that the primary observed effect is not an artifact of a multisample measurement. The single-crystal measurement has a flatter character than the stack measurement; this could be due to slight misalignments in the stack, a smaller number of domains in one crystal as opposed to a stack of 30 , or a stronger influence of the background on a smaller signal, to name a few possibilities. The single crystal was a part of the measured stack; it was chosen from the stack because it was the thickest, at about $0.2 \mathrm{~mm}$.

\section{High-field magnetization with more iron}

Pulsed field measurements of $\mathrm{Fe}_{x} \mathrm{NbS}_{2}$ with $x=0.339$ (slightly higher iron content than the perfect $x=1 / 3$ ) are sim- ilar to those of the main sample studied in the text, $x=0.330$, with plateaus in the magnetization appearing at low temperature (Fig. 11). The exact locations of the transitions differ from the locations found in the main $x=0.330$ sample, indicating a sensitive dependence of the phase boundary location on the exact iron content. It should be noted that this pulsed field data on the $x=0.339$ sample is not background corrected (a procedure which requires a second magnet pulse to go along with each measurement), and so its precise shape may differ slightly from what is presented.

\section{Torque magnetometry}

The anisotropy of the magnetic response of $\mathrm{Fe}_{x} \mathrm{NbS}_{2}$ was further studied via torque magnetometry. The full data set is given in Fig. 12(a). The measurement was performed with 

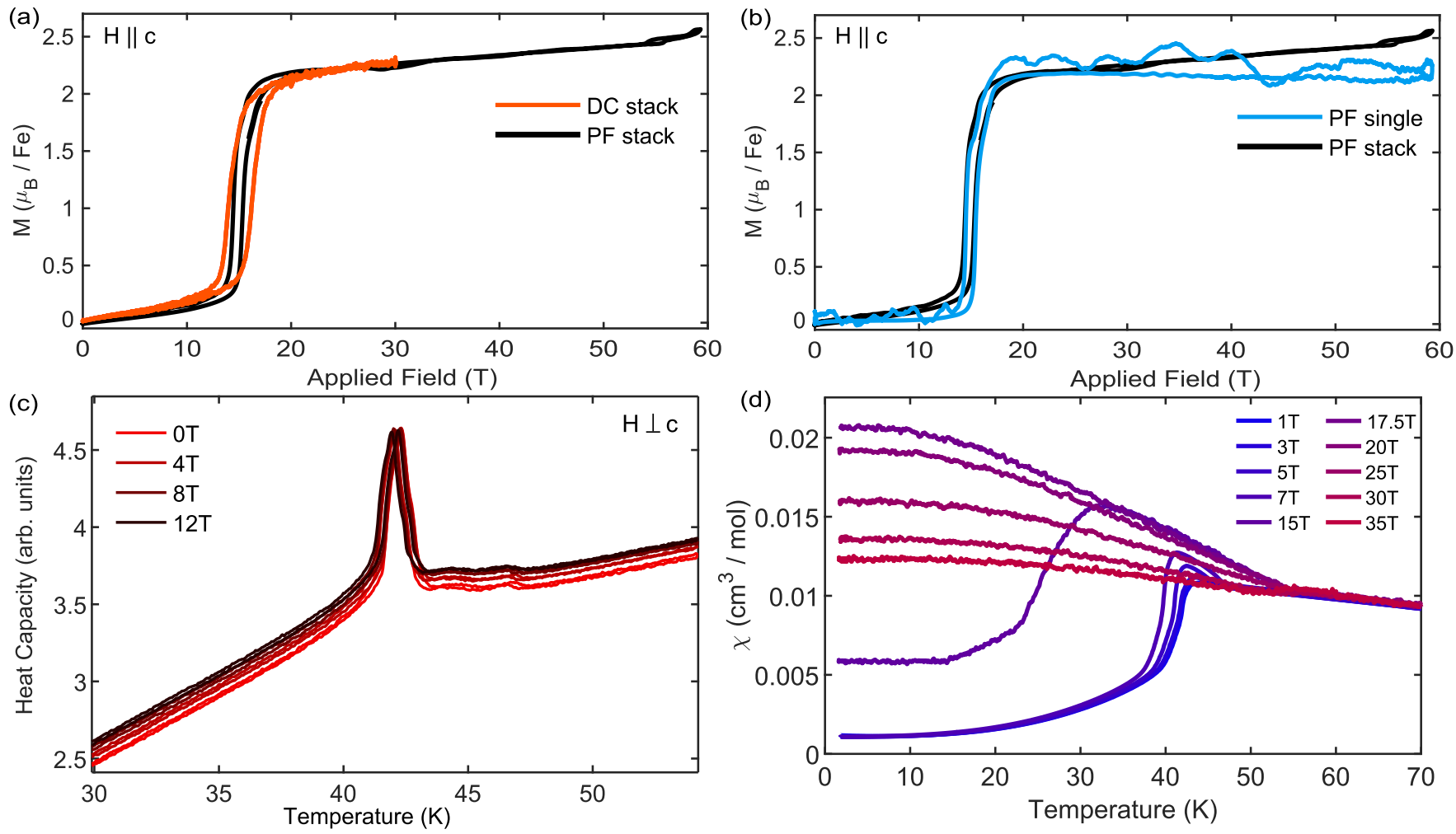

FIG. 13. (a) Comparison of the magnetization measured in 30-T DC field to the magnetization measured in 60-T pulsed field (PF). The DC measurement was taken at $1.6 \mathrm{~K}$, and the PF measurement shown was taken at $4 \mathrm{~K}$. (b) Comparison of PF measurement of a single crystal to PF measurement of the stack of crystals used for the primary results of this paper. Both measurements were taken at $4 \mathrm{~K}$. (c) Heat capacity with an in-plane applied magnetic field. (d) Susceptibility as a function of temperature measured in fields ranging from 1 to $35 \mathrm{~T}$.

a magnetic field applied at various angles $\theta$ with respect to the sample's $c$ axis, as shown in Fig. 12(b). For each temperature and field, the torque as a function of angle $\theta$ was fit to an equation of the form $\tau=Q_{1} \sin (2 \theta)+Q_{2} \sin (4 \theta)$, as illustrated in Fig. 12(c). These components were then analyzed as a function of temperature at each field in order to identify phase transitions [Figs. 12(d) and 12(e)]. The two strongest features correspond to the lower temperature and intermediate-temperature phase boundaries shown in the phase diagram in the main text. Notably, these features are identified using different components of the torque response.

\section{In-plane heat capacity}

While the transitions move dramatically with the application of field along the $c$ axis [Fig. 2(b) in the main text], magnetic field applied perpendicular to the $c$ axis has no effect on the heat capacity or on the transitions that it probes, as seen in Fig. 13(c).

\section{Susceptibility vs temperature}

The phase transitions discussed in the main text are also visible in DC field measurements taken as a function of temperature, shown in Fig. 13(d).

\section{Additional intermediate phase}

The main text focuses on the dominant features seen in the heat capacity and magnetization. However, there is also a minor peak visible in both measurements, shown in magnetization in Fig. 14. We ascribe this feature to an intermediate phase between the stripy and plateau phases. However, since the corresponding peaks are small, it is not possible to track the transitions with the same level of certainly as the dominant phases. Nevertheless, the intermediate phase seems to have magnetization of around $1.6 \mu_{B} / F e$, which is almost exactly $1 / 3$ of the saturation magnetization. This is suggestive of a connection to UUD spin configurations in a three-spin unit cell, which are responsible for $1 / 3$ magnetization plateaus.

\section{High field transport}

Transport measurements taken in 60-T pulsed field are shown in Fig. 15. These measurements show increased scattering at the primary phase boundaries, as seen in the magnetoresistance, as well as a change in slope of the Hall resistance over the transition.

\section{Nuclear magnetic resonance}

Nuclear magnetic resonance (NMR) measurements were used to study the magnetic state at fields below and near the plateau phase. The iron exchange field is studied via its effect on the ${ }^{93} \mathrm{Nb}$ lattice (with nuclear spin $I=9 / 2$, $\gamma=10.405 \mathrm{MHz} / \mathrm{T})$. NMR measurements are shown in Figs. 16(a) and 16(b), centered close at 7.16 and $15.38 \mathrm{~T}$, respectively. In both field ranges, the nine peaks expected from niobium in the paramagnetic regime are seen at $100 \mathrm{~K}$, 
(a)

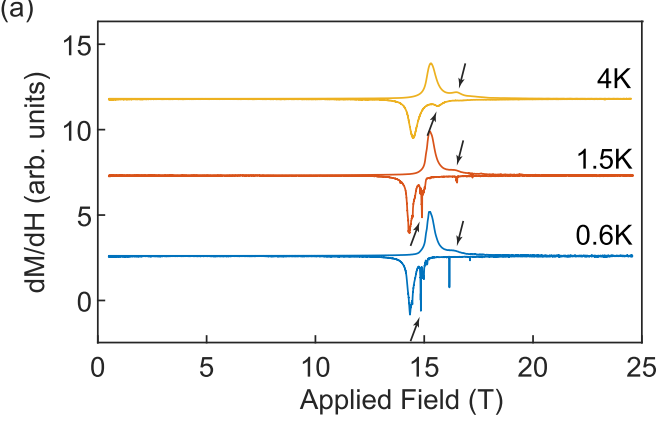

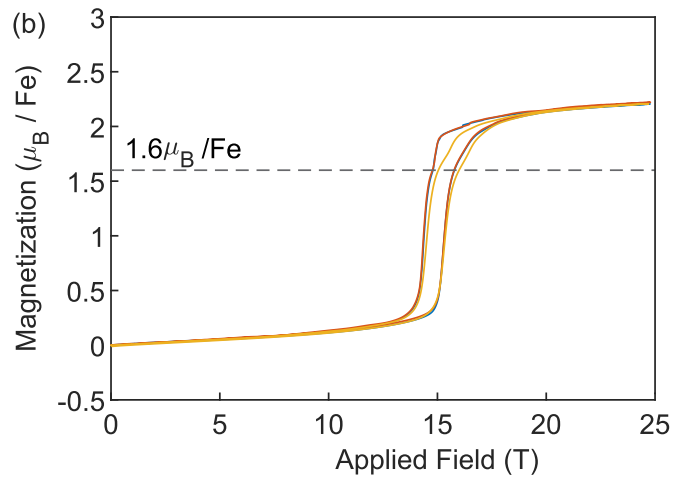

FIG. 14. (a) Susceptibility measured at $4,1.5$, and $0.6 \mathrm{~K}$, offset for discernibility. Small features corresponding to intermediate phase boundary are indicated with black arrows. (b) Magnetization at these temperatures, with dotted line indicating $1.6 \mu_{B} / F e$, which is approximately $1 / 3$ of the saturation magentization.

and at low temperatures, two broad peaks indicative of a long-range AFM order with only two predominant spin orientations. It is important to note that the $4.2-\mathrm{K}$ measurement was performed moving from high field to low, corresponding
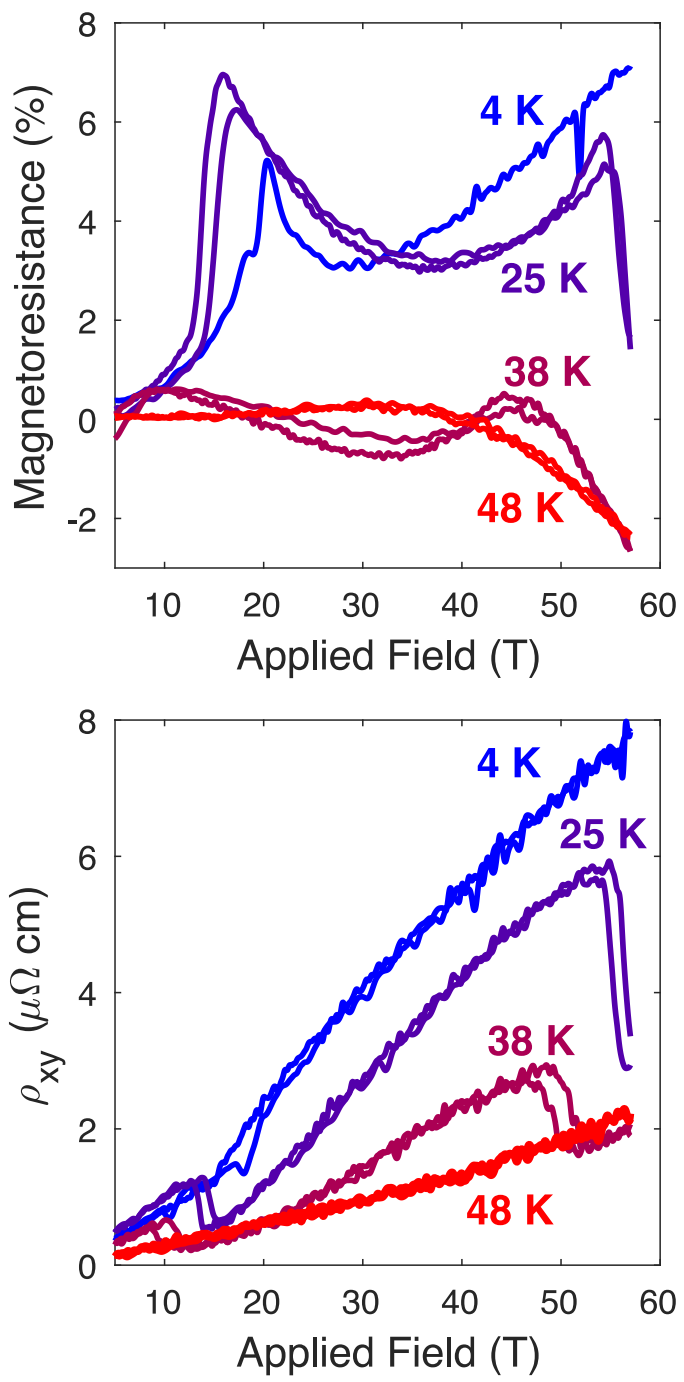

FIG. 15. Magnetoresistance and Hall resistance measured in high pulsed field. to the upper branch of the hysteresis seen in the magnetization, Fig. 16(c). While the system moves partially through a transition in the high-field measurement, Fig. 16(b), the shape of the NMR signal remains comparable to that in the low-field stripe phase in that there continue to be two predominant local field strengths, and therefore only two spin orientations. While the (a)

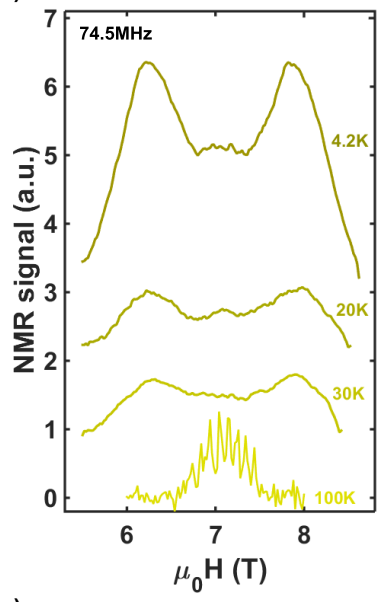

(b)

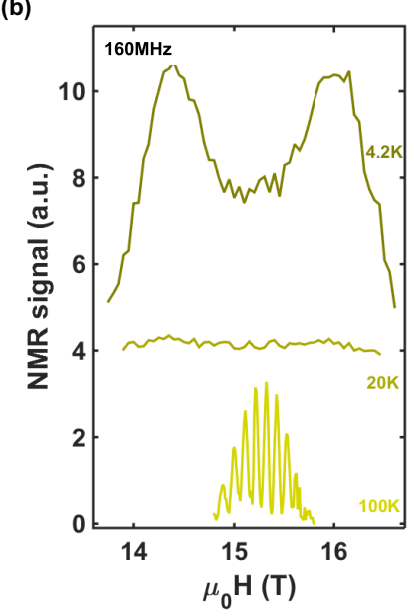

(c)

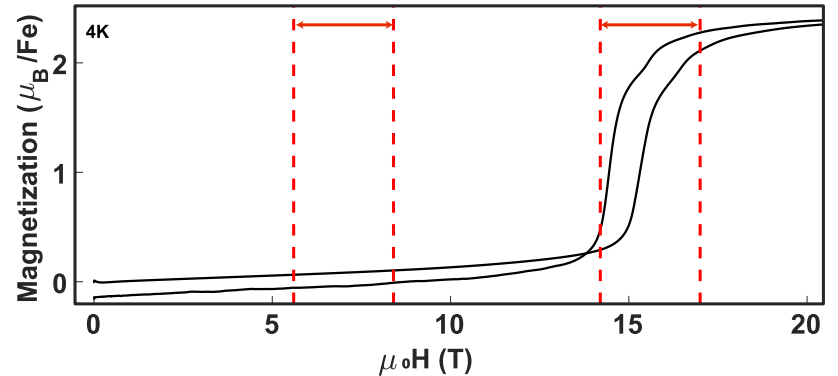

FIG. 16. Field-swept NMR spectra for several temperatures from 100 to $4.2 \mathrm{~K}$, with the magnetic field applied along the $c$ axis of the samples. (a) Spectra measured at $74.5 \mathrm{MHz}$, centered close to $7 \mathrm{~T}$. (b) Spectra measured at $160 \mathrm{MHz}$, centered slightly above $15 \mathrm{~T}$. (c) The magnetization as a function of field at $4 \mathrm{~K}$, with the regions probed by NMR indicated explicitly. The branches of the hysteresis do not match up perfectly due to a gain saturation issue during the pulsed field measurement. 
plateau state could hypothetically be quite complicated, the findings from NMR suggest that the plateau phase retains only two spin orientations. This is consistent with our treatment, which has considered a range of magnetic until cell sizes and determined that a relatively simple spin structure is best suited to explain our other measurements. Notably the peak in Fig. 16(a) are symmetric, whereas the peaks in Fig. 16(b) are slightly asymmetric, consonant with a higher occupation of one spin orientation as expected of an UUUD phase.

\section{APPENDIX C: DETAILS OF OUR DFT CALCULATIONS}

For our first-principles density functional theory (DFT) calculations, we employ the Vienna $a b$ intitio simulation package (VASP) [39] and use the generalized gradient approximation (GGA) of Perdew-Burke-Ernzerhof (PBE) [30] and the projector augmented-wave (PAW) method [40]. All calculations are spin polarized and fully relativistic, including self-consistent spin-orbit interactions. To approximately account for the localized nature of the $d$ electrons on the Fe atoms, we add a Hubbard $U$ correction $(\mathrm{GGA}+\mathrm{U})$ [31]. We use the rotationally invariant version of GGA $+U$ by Dudarev et al. [41] and select $U_{\text {eff }}=U-J=0.3 \mathrm{eV}$ for the Fe $d$ states. Our calculations are performed using the experimental lattice constants $a=5.76 \AA$ and $c=12.20 \AA$ with experimental atomic coordinates [7]. As mentioned in the main text, this $U$ value is chosen so that the $\mathrm{PBE}+\mathrm{U}$ value of the magnetocrystalline anisotropy $\mathrm{D}$, at the experimental lattice parameters, matches with our Curie-Weiss estimate of $D \approx 1 \mathrm{meV}$. As an aside, we calculate $\mathrm{D}$ by comparing selfconsistent total energies in the case of all $\mathrm{Fe}^{2+}$ spins aligned along the $a$ ([100]) axis and along the $c([001])$ axis:

$$
D=\frac{E_{[100]}-E_{[001]}}{2 S^{2}}
$$

where we use $S=2$ for the Fe spins. (Our PBE $+\mathrm{U}$ value for the effective Fe moment is $3.1 \mu B$ with $U=0.3 \mathrm{eV}$ for the AFM stripe ground state, somewhat smaller than but consistent with the ideal pure-spin $4 \mu B$ limit corresponding to $S=2$ ). The factor of 2 accounts for the two Fe atoms in the primitive unit cell. We use a $650-\mathrm{eV}$ cutoff for our plane-wave basis set, with a $\Gamma$-centered $k$-point mesh of $12 \times 12 \times 6$ for the primitive two-Fe spin unit cell of $\mathrm{Fe}_{1 / 3} \mathrm{NbS}_{2}$. For the $1 \times \sqrt{3} \times 1$ and $2 \times \sqrt{3} \times 1$ supercells used to generate four out of the six inequivalent magnetic configurations needed to calculate the exchange couplings, we use reduced meshes of $12 \times 7 \times 6$ and $6 \times 7 \times 6$ respectively. All above parameters lead to total energies converged to less than $1 \mathrm{meV}$ per $\mathrm{Fe}$ atom.

Finally, we elaborate on the apparent overestimation by $\mathrm{PBE}+\mathrm{U}$, for our choice of $\mathrm{U}$, of the Heisenberg exchange constants $\left(J_{1}, J_{2}, J_{1 c}, J_{2 c}\right)$, as discussed in the main text. To test whether we might get smaller exchange constants by increasing $U$ while still obtaining a reasonable anisotropy, we calculated $D$ for a range of $U$ values, and additionally examined the effect of including a separate Hund's on-site exchange $J$ as in the method of Lichtenstein et al. [42]. We find that with $J=0, U>2 \mathrm{eV}$ yields the incorrect sign of $D$ (i.e., the preferred orientation of spins is incorrectly predicted to be in the $a b$ plane rather than along $c$ ); thus, more typical, larger values of $U$ yield a a qualitatively incorrect anisotropy as compared to experiment for $\mathrm{Fe}_{1 / 3} \mathrm{NbS}_{2}$. Including the Hund's exchange and for the set of parameters $U=3, J=1.2 \mathrm{eV}$ we also obtain a reasonable $D=1.2 \mathrm{eV}$. However, with these values of $U$ and $J$ the relative couplings change qualitatively and the calculated ground state becomes FM within a single Fe plane, as opposed the the stripy AFM state predicted experimentally. For these reasons, we settled on $U_{\text {eff }}=0.3 \mathrm{eV}$ in this work.
[1] T. Jungwirth, J. Sinova, A. Manchon, X. Marti, J. Wunderlich, and C. Felser, Nat. Phys. 14, 200 (2018).

[2] M. Takigawa and F. Mila, Magnetization plateaus, in Introduction to Frustrated Magnetism: Materials, Experiments, Theory, edited by C. Lacroix, P. Mendels, and F. Mila (Springer, Berlin, 2011), pp. 241-267.

[3] N. L. Nair, E. Maniv, C. John, S. Doyle, J. Orenstein, and J. G. Analytis, Nat. Mater. 19, 153 (2020).

[4] J. Zelezny, H. Gao, K. Vyborny, J. Zemen, J. Masek, A. Manchon, J. Wunderlich, J. Sinova, and T. Jungwirth, Phys. Rev. Lett. 113, 157201 (2014).

[5] P. Wadley, B. Howells, J. Zelezny, C. Andrews, V. Hills, R. P. Campion, V. Novák, K. Olejník, F. Maccherozzi, S. S. Dhesi, S. Y. Martin, T. Wagner, J. Wunderlich, F. Freimuth, Y. Mokrousov, J. Kunes, J. S. Chauhan, M. J. Grzybowski, A. W. Rushforth, K. W. Edmonds, B. L. Gallagher, and T. Jungwirth, Science 351, 587 (2016).

[6] S. Mankovsky, S. Polesya, H. Ebert, and W. Bensch, Phys. Rev. B 94, 184430 (2016).

[7] B. Van Laar, H. M. Rietveld, and D. J. W. Ijdo, J. Solid State Chem. 3, 154 (1971).
[8] Y. Suzuki, T. Ikeda, S. Richardson, and J. W. Yamaguchi, in Proceedings of the Fifth International Symposium on Advanced Nuclear Energy Research (Japan Atomic Energy Research Institute, Mito, Japan, 1993), pp. 343-346.

[9] A. Little, C. Lee, C. John, S. Doyle, E. Maniv, N. L. Nair, W. Chen, D. Rees, J. W. F. Venderbos, R. M. Fernandes, J. G. Analytis, and J. Orenstein, Nat. Mater. 19, 1062 (2020).

[10] L. Seabra and N. Shannon, Phys. Rev. B 83, 134412 (2011).

[11] M. Ye and A. V. Chubukov, Phys. Rev. B 96, 140406(R) (2017).

[12] M. Ye and A. V. Chubukov, Phys. Rev. B 95, 014425 (2017).

[13] T. Ono, H. Tanaka, H. Aruga Katori, F. Ishikawa, H. Mitamura, and T. Goto, Phys. Rev. B 67, 104431 (2003).

[14] L. E. Svistov, A. I. Smirnov, L. A. Prozorova, O. A. Petrenko, L. N. Demianets, and A. Y. Shapiro, Phys. Rev. B 67, 094434 (2003).

[15] A. Wiedenmann, L. Regnault, P. Burlet, J. Rossat-Mignod, O. Koundé, and D. Billerey, J. Magn. Magn. Mater. 74, 7 (1988).

[16] Y. Shirata, H. Tanaka, T. Ono, A. Matsuo, K. Kindo, and H. Nakano, J. Phys. Soc. Jpn. 80, 093702 (2011).

[17] M. V. Gvozdikova, P.-E. Melchy, and M. E. Zhitomirsky, J. Phys.: Condens. Matter 23, 164209 (2011). 
[18] T. Susuki, N. Kurita, T. Tanaka, H. Nojiri, A. Matsuo, K. Kindo, and H. Tanaka, Phys. Rev. Lett. 110, 267201 (2013).

[19] J. Alicea, A. V. Chubukov, and O. A. Starykh, Phys. Rev. Lett. 102, 137201 (2009).

[20] A. I. Coldea, L. Seabra, A. McCollam, A. Carrington, L. Malone, A. F. Bangura, D. Vignolles, P. G. van Rhee, R. D. McDonald, T. Sörgel, M. Jansen, N. Shannon, and R. Coldea, Phys. Rev. B 90, 020401(R) (2014).

[21] O. Gorochov, A. L. Blanc-soreau, J. Rouxel, P. Imbert, and G. Jehanno, Philos. Mag. B 43, 621 (1981).

[22] M. Sundararajan, A. Narayanasamy, T. Nagarajan, C. Sunandana, G. S. Rao, D. Niarchos, and G. Shenoy, J. Phys. Chem. Solids 44, 773 (1983).

[23] S. Doyle, C. John, E. Maniv, R. A. Murphy, A. Maniv, S. K. Ramakrishna, Y.-L. Tang, R. Ramesh, J. R. Long, A. P. Reyes, and J. G. Analytis, Tunable giant exchange bias in an intercalated transition metal dichalcogenide, arXiv:1904.05872 [cond-mat.str-el].

[24] F. Hulliger and E. Pobitschka, J. Solid State Chem. 1, 117 (1970).

[25] K. Anzenhofer, J. Van Den Berg, P. Cossee, and J. Helle, J. Phys. Chem. Solids 31, 1057 (1970).

[26] N. Doi and Y. Tazuke, J. Phys. Soc. Jpn. 60, 3980 (1991).

[27] R. H. Friend, A. R. Beal, and A. D. Yoffe, Philos. Mag. 35, 1269 (1977).

[28] P. Sengupta, C. D. Batista, R. D. McDonald, S. Cox, J. Singleton, L. Huang, T. P. Papageorgiou, O. Ignatchik,
T. Herrmannsdörfer, J. L. Manson, J. A. Schlueter, K. A. Funk, and J. Wosnitza, Phys. Rev. B 79, 060409(R) (2009).

[29] D. C. Johnston, Phys. Rev. B 95, 094421 (2017).

[30] J. P. Perdew, K. Burke, and M. Ernzerhof, Phys. Rev. Lett. 77, 3865 (1996).

[31] J. P. Perdew and W. Yue, Phys. Rev. B 33, 8800(R) (1986).

[32] I. Yang, S. Y. Savrasov, and G. Kotliar, Phys. Rev. Lett. 87, 216405 (2001).

[33] E. Bousquet and N. Spaldin, Phys. Rev. B 82, 220402(R) (2010).

[34] C. Lee, J. Hong, W. J. Son, E. Kan, J. H. Shim, and M. H. Whangbo, RSC Adv. 6, 22722 (2016).

[35] C. Loschen, J. Carrasco, K. M. Neyman, and F. Illas, Phys. Rev. B 75, 035115 (2007).

[36] R. L. Martin and F. Illas, Phys. Rev. Lett. 79, 1539 (1997).

[37] T. Linneweber, J. Bünemann, U. Löw, F. Gebhard, and F. Anders, Phys. Rev. B 95, 045134 (2017).

[38] M. Singh, J. Callaway, and C. Wang, Phys. Rev. B 14, 1214 (1976).

[39] G. Kresse and J. Furthmuller, Phys. Rev. B 54, 11169 (1996).

[40] P. E. Blochl, Phys. Rev. B 50, 17953 (1994).

[41] S. L. Dudarev G. A. Botton, S. Y. Savrasov, C. J. Humphreys, and A. P. Sutton, Phys. Rev. B 57, 1505 (1998).

[42] A. I. Liechtenstein, V. I. Anisimov, and J. Zaanen, Phys. Rev. B 52, R5467(R) (1995). 